\title{
Modelling and Simulation of 3 Blade Helicopter's Rotor Model
}

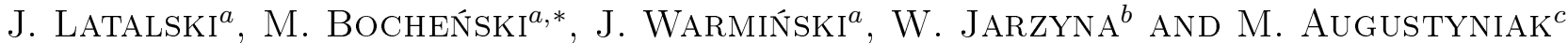 \\ ${ }^{a}$ Department of Applied Mechanics, Lublin University of Technology, Lublin, Poland \\ ${ }^{b}$ Department of Electrical Drive Systems and Electrical Machines, Lublin University of Technology, Lublin, Poland \\ ${ }^{c}$ Induster Sp. z o.o., Lublin, Poland
}

\begin{abstract}
Results of model tests of a rotating composite beam with integrated piezoelectric active element are presented in the paper. A proposed electromechanical system is a simplified model of the structure of a modern helicopter rotor blade. Numerical analysis of the considered system is developed by means of the finite element method. In addition, the laboratory setup has been built in order to perform real experimental studies. Selected static and dynamic characteristics of the object are determined by a series of numerical simulations. The results are compared with the outcomes of tests performed on the experimental setup. A very good agreement between numerical simulation and experiment results is observed.
\end{abstract}

DOI: 10.12693 /APhysPolA.125.1380

PACS: 02.70.Dh, 07.05.Tp, 45.20.dc, 46.70.De, 77.65.-j, 77.84.Cg, 77.84.Lf

\section{Introduction}

Dynamic and control of the rotating beam structures with embedded electrically or magnetically induced strain actuators are intensively studied in recent years. Implementation of active elements integrated to the hosting structure opens new promising perspectives of enhancing system's characteristics and provides the means for efficient control of its dynamic properties. To take the maximum benefit of these measures and full potential of the actuating element an in depth knowledge about multi-physics rotor dynamic properties is requested. It is also of primary importance for active controlled systems in view of e.g. proper control algorithms choice or actuator positioning.

This work is focused on numerical and experimental examination of a rotating composite beam with integrated piezoelectric active element. A simple case of dynamic analysis involving piezoelectric and structural coupling is performed. In the carried out tests most classical and non-classical effects observed in beam-like structures are taken into account and examined e.g. in [1]. Among others these include composite material anisotropy, transverse shear deformation and cross-section warping, as well as an arbitrary beam presetting angle. Moreover, the possible interactions between various natural modes of vibrations as suggested in previous papers $[2,3]$ are considered.

For comparative studies a laboratory test stand is prepared. The setup enables the blades to be mounted to the hub at an arbitrary pitch angle. The buildup DC motor provides precise hub's speed control.

The outcomes of finite element method numerical simulations are compared to laboratory experimental results.

*corresponding author; e-mail: m.bochenski@pollub.pl

\section{FE modeling and numerical results}

The finite element analysis (FEA) technique provides large flexibility and allows study of complex electrical and mechanical systems. After initial research in late ' 80 and early '90 e.g. [4] fully electromechanical coupled piezoelectric elements became available in commercial finite element code packages [5]. Later on several benchmark examples and testing techniques were proposed to validate the numerical models of piezoelectric materials and electromechanical structures [6, 7].

Nowadays a finite element simulation is a standard procedure in electromechanical systems analysis and design. Two packages (ANSYS by Ansys Inc. and ABAQUS by Simulia) seem to be most common ones. The analysis performed in this research has been done in ABAQUS system.

At the very first stage of numerical research the finite element model of the self-contained piezoelectric actuator has been prepared. For numerical and later laboratory tests, M-8503-P2 element made by Smart Material Corp., Sarasota (FL) USA has been used. This is a piezoelectric transducer of $d_{31}$ effect type. Despite of a modular structure of the transducer under consideration (multiple sections of electro-couples distant by $0.5 \mathrm{~mm}$ from each other) a supplementary body made of orthotropic, homogeneous piezoelectric material has been proposed, where voltage has been applied to opposite specimen faces (upper and lower ones). The transducer's domain has been modeled in ABAQUS package by the solid continuum elements type C3D20E, i.e. 20-nodal second order, having four degrees of freedom in each node (three translational DOF and one for electric charge). Additional prerequisite equilibrium constraints on nodal electric charge have been set to ensure uniform electric potential distribution over the electrodes' surfaces.

The initial tests have shown that the originally given by the manufacturer value of the piezoelectric constant $d_{31}$ equal to $-2.1 \times 10^{2} \mathrm{pC} / \mathrm{N}$ specific for the transducer's 
electro-couples needed updating. To meet the actuator performance data a series of further simulations has been run to estimate an effective value of the transducer's $d_{31}$ constant.

More specific information on piezoelectric Macro Fiber Composite transducers modeling by means of supplementary orthotropic bodies is given in Ref. [8]; some further comments on multi-domain FE models can be found in e.g. [9].

Verification of the elaborated piezoelectric element model has been done by means of two tests - free strain tests and blocking force test. Figure 1 presents the outcomes the simulation after FE model fine-tuning. A very good agreement between FEM results and specimen's characteristics as given by manufacturer is noticeable.

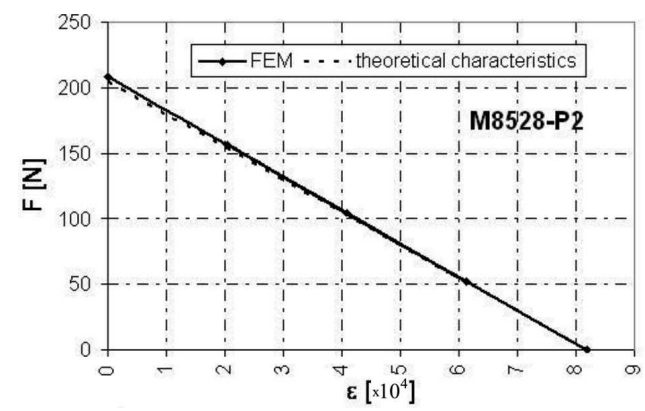

Fig. 1. MFC M8528-P2 strain vs. force plot - comparison of FEM results and manufacturer data.

The verified model of the piezoelectric transducer has been used to simulate the composite cantilever beam with M-8503-P 2 active element. To maximize the effectiveness of the transducer bending mode it has been bonded on the upper face of a specimen, directly at the clamped end (see Fig. 2). In numerical model this effect has been achieved by TIE constraints method, which results in joining appropriate mechanical degrees of freedom (DOF) of both bodies (transducer and master beam).

The hosting structure (master beam) is made of glass-epoxy unidirectional composite prepreg TVR 380 600 M12 26\% R-glass material. The fibers orientation in the specimen's subsequent layers is as follows: $45^{\circ} /-45^{\circ} / 90^{\circ} / 90^{\circ} /-45^{\circ} / 45^{\circ}$ with respect to an axis pointing along the beam length. Numerical model of the beam has been defined as a lamina type one. This approach enables modeling of the composite specimens as a set of orthotropic layers in plane-stress state. In performed numerical simulations the following data provided by the manufacturer of the composite have been used: Young modulus along fibers $E_{1}=56000 \mathrm{MPa}$, transversal Young modulus $E_{2}=16000 \mathrm{MPa}$, shear moduli $G_{12}=4050 \mathrm{MPa}, G_{13}=G_{23}=4000 \mathrm{MPa}$ and Poisson's ratio $v_{12}=0.4$. The ABAQUS finite element model based on shell elements has been made according to Layup-ply technique. In the performed analysis S8R elements have been applied - i.e. second order ones with reduced integration.

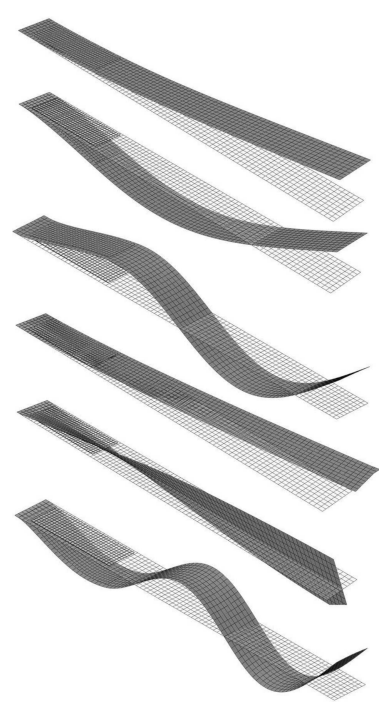

Fig. 2. First six natural mode shapes of a beam-transducer specimen extracted from ABAQUS software.

At the next stage of analysis simulation tests in dynamic conditions have been run. Influence of systems' rotational speed and blade's pitch angle on the natural frequencies value has been examined. For comparative studies cases of open and closed poles of actuator's electric circuit have been run. Based on obtained numerical results Campbell diagrams have been prepared (natural frequency versus rotation speed).

Next, the natural frequencies and the mode shapes for static (rotationless) system have been extracted. For the purposes of further analysis first six modes have been chosen (Fig. 2). These have been four flapwise bending modes (flexible plane), torsion and chordwise bending (stiff plane) mode.

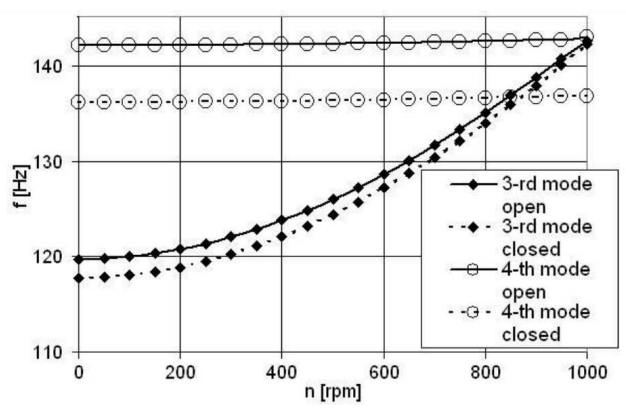

Fig. 3. Campbell diagram - interaction between 3rd and 4 th modes $\left(0^{\circ}\right.$ presetting angle).

For all flapwise bending modes the increase of natural frequencies due to stiffening effect caused by system's rotational speed is clearly observed. Frequency for first chordwise bending mode (4th mode, see Fig. 2) is almost independent of rotational speed (see Fig. 3). Surprisingly, for first torsion mode and $45^{\circ}$ beam pitch an- 


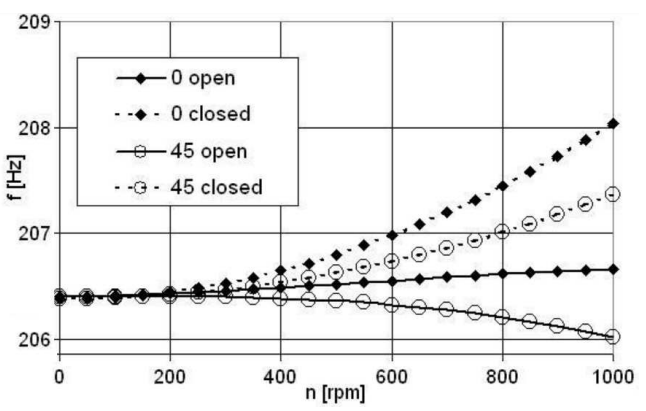

Fig. 4. Campbell diagram -5 th mode (first torsion mode).

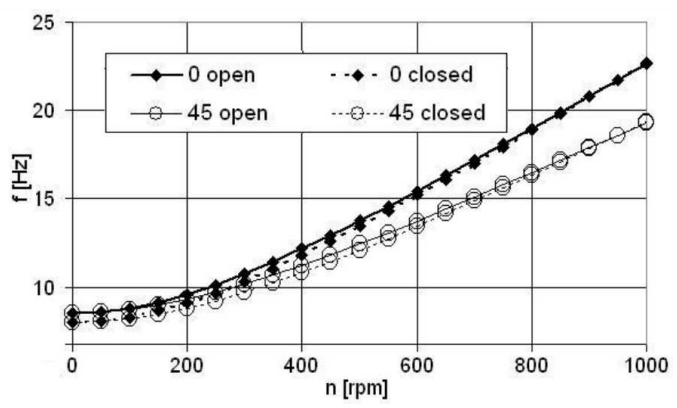

Fig. 5. Campbell diagram - first bending mode for $0^{\circ}$ and $45^{\circ}$ presetting angle.

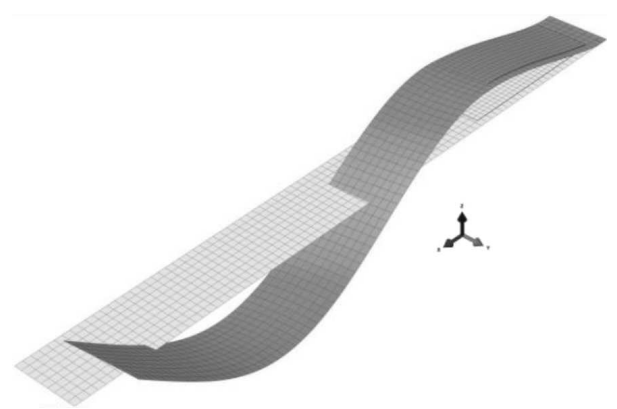

Fig. 6. Modes coupling - interaction between 3rd and 4 th mode $(1000 \mathrm{rpm})$.

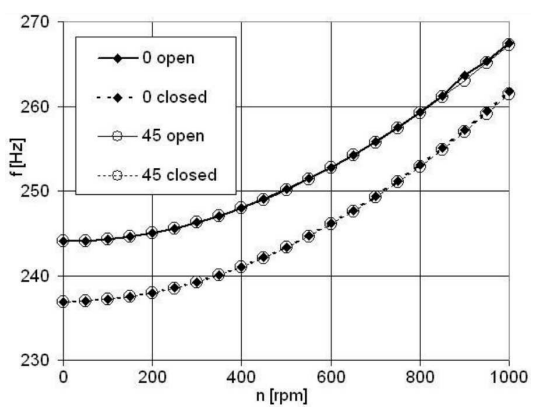

Fig. 7. Campbell diagram -6 th mode. gle a small softening effect is visible (Fig. 4). For all modes except torsion closing of electric circuit decreases the values of natural frequency but for lower modes this phenomenon is less evident along to rotational speed. Influence of pitch angle on natural frequencies is well visible for lower bending modes, especially at higher rotational speeds (see Fig. 5). Moreover, for $1000 \mathrm{rpm}$ hub speed strong interaction (coupling) between $3 \mathrm{rd}$ and 4 th mode is observed. This corresponds to the fact that natural frequencies of these two modes become equal (Figs. 3, 6). For higher modes (Fig. 7) influence of blade's pitch angle on the natural frequencies is negligible but strong influence of electric circuit state (open/closed) for all range of hub speeds is observed.

\section{Experimental setup and results}

The prepared laboratory setup consists of three blades made of glass-epoxy composite material mounted on a hub. Construction of the hub allows fixing the blades at an arbitrary presetting (pitch) angle (Fig. 8). The hub of the rotor is driven by the DC motor system with pulse width modulation (PWM) speed control. Macro fiber composite (MFC) elements embedded onto rotating beams are used as actuators, but can also be used as strain gauges (sensors) [10]. The power supply to the actuators is provided by means of an on-hub mounted electronic device.

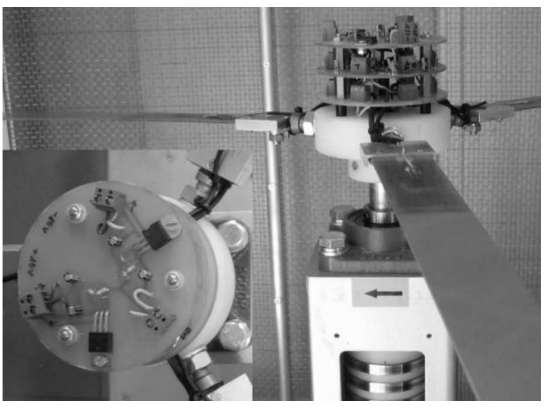

Fig. 8. Experimental setup.

At the first stage of experimental tests static characteristics of the laboratory electromechanical system have been measured and compared to finite element simulation results. As presented in Fig. 9, numerical and experimental values of clamped beam's free end deflection versus vertical force acting at this point proved to be satisfactory close.

Next, influence of rotation speed and pitch angle on the blade's response has been tested. To this end time series signal from strain gauge fast Fourier transform (FFT) has been recorded. It is observed that in spite of constant rotating speed the response of the blade is polyharmonic.

Along with pitch angle increase the more complex dynamic is to be noted (Fig. 10). For example, for $40^{\circ}$ pitch angle new harmonics, that are not present for $0 / 20^{\circ}$ settings appear (Fig. 10, e.g. $3.3 \mathrm{~Hz}$ frequency). Although 


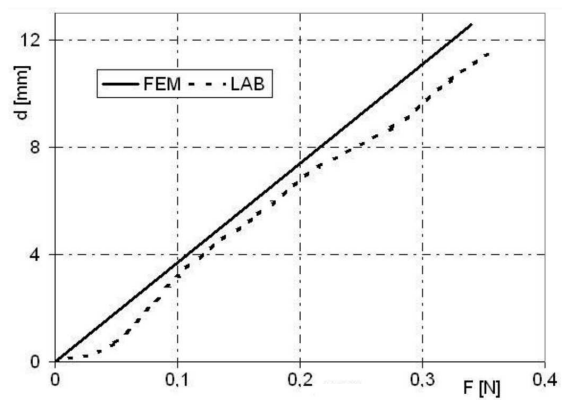

Fig. 9. Beam's free end deflection versus force.

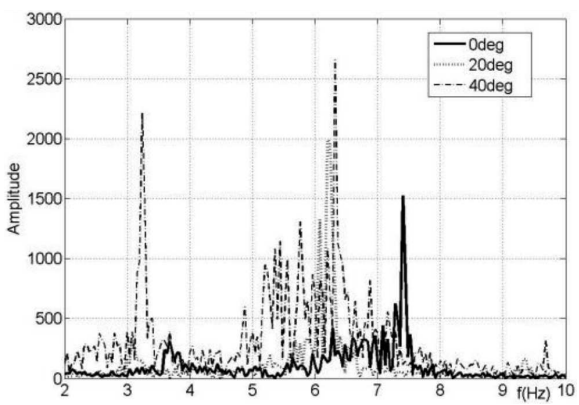

Fig. 10. Influence of pitch angle (200 rpm).

for lower settings (e.g. $20^{\circ}$ ) only a slight shift in frequencies is observed if compared to $0^{\circ}$ pitch angle.

For constant pitch angle along with rotation speed change response spectra shifts to the right direction (Fig. 11). Then, stiffening effect due to centrifugal force observed in numerical simulations is confirmed.

\section{Conclusions}

Finite element and experimental tests of a rotating composite beam with integrated piezoelectric MFC active element have been performed. A series of simulation tests were carried out and selected static and dynamic characteristics of the object were determined. Good agreement of numerical and laboratory results has validated the numerical modeling approach.

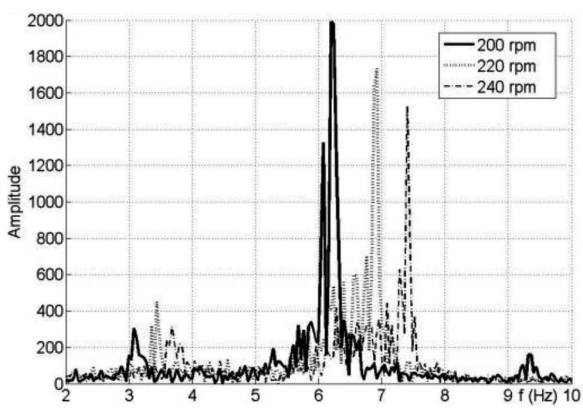

Fig. 11. Influence of rotation speed $\left(20^{\circ}\right)$.
More detailed analysis of the results has shown that the open/closed state of transducer's electric circuit have a significant influence on system's dynamic. Therefore, a simple on/off control of the considered rotor design seems to be possible. However, the significance of these phenomena for lower modes decays as the hub rotational speed increase. In opposite to this observation, for higher modes this relation is noticed in the full range of tested rotational speeds.

It is observed that the impact of a pitch angle on system's response gets more evident along with hub speed increase.

For all flapwise bending modes (flexible plane) the stiffening effect due to centrifugal force is evidently observed. For e.g. 1000 rpm hub speed strong coupling between both bending modes is observed.

\section{Acknowledgments}

The financial support of Structural Funds in the Operational Programme - Innovative Economy (IE OP) financed from the European Regional Development Fund - Project "Modern material technologies in aerospace industry", No. POIG.01.01.02-00-015/08-00 is gratefully acknowledged.

Financial support of Development of Young Researchers Program 30/MN/2012 is gratefully acknowledged by the second author.

\section{References}

[1] F. Georgiades, J. Latalski, J. Warmiński, Trans. Inst. Aviation 218, 36 (2011).

[2] J. Latalski, F. Georgiades, J. Warmiński, J. Phys., Conf. Series 382, 012021 (2012).

[3] L. Librescu, O. Song, Appl. Mech. Rev. 44, 174 (1991).

[4] W.-S. Hwang, H.C. Park, AIAA J. 5, 31 (1993).

[5] M.W. Lin, A.O. Abatan, C.A. Rogers, J. Intellig. Mater. Syst. Struct. 6, 5 (1994).

[6] M.C. Reaves, L.G. Horta, in: 42nd AIAA/ASME/ASCE/ASC Struct. Struct. Dynam. Mater. Conf., AIAA, Reston 2001, paper 2001-1466.

[7] P.C. Dumir, S. Kapuria, P. Kumari, J.K. Nath, ZAMM J. Appl. Math. Mechan. 1, 88 (2008).

[8] J. Latalski, Maintenance and Reliability 4, 72 (2011).

[9] B.T. Wang, P.H. Chen, R.-L. Chen, J. Mechan. 2, 22 (2006).

[10] W. Jarzyna, M. Augustyniak, J. Warmiński, M. Bocheński, Przeglad Elektrotechniczny 7, 320 (2010) (in Polish). 